\title{
A histopathological study of the kidneys in a selected group of children with primary nephrotic syndrome
}

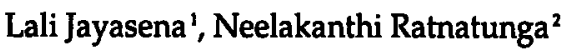 \\ The Ceylon Journal of Medical Science 1998; 41: 19-24
}

\begin{abstract}
22 children out of a cohort of 63 admitted to the Teaching Hospital, Peradeniya with primary nephrotic syndrome were subjected to percutaneous renal biopsy. Renal biopsies were performed on:

- children with primary nephrotic syndrome who were steroid sensitive but developed frequent relapses and were found to be steroid dependant,

- children resistant to steroids from the onset of the illness, and

- children who presented at the onset with atypical features such as persistent hematuria and/or hypertension.
\end{abstract}

Paraffin sections of the renal biopsies were stained with haemotoxylin and eosin, PAS and methenamine silver stain. Immunohistochemical staining for IgG, IgM, IgA and C 3 was also done. Thirteen out of the 22 children biopsied had significant structural changes on histopathological study. Seven of the 13 patients showed focal segmental glomerulosclerosis. Six out of the nine children who showed minimal change histology were females which was in contrast to the usual male preponderance seen for this lesion. Since the renal biopsies were done only in patients with diagnostic or therapeutic problems it is possible that some of the patients who were categorised under minimal change pathology may subsequently turn out to be focal segmental glomerulosclerosis as these lesions are focal.

An attempt was also made to correlate the clinical features to the glomerular pathology.
This is a preliminary report of an ongoing study and it is too early to comment on the outcome of these patients.

\section{Introduction}

Several studies from most parts of the world (1-5) except Africa have shown that the majority of children with primary nephrotic syndrome have minimal change histology on renal biopsy and that most of them are responsive to steroids.

In contrast to the rest of the world the nephrotic syndrome in children from tropical Africa was fund to be due to Plasmodium malariae (6). A study from South Africa reported that $86 \%$ of South African children had significant structural lesions in their kidneys and were unresponsive to steroid therapy. It was emphasised that those lesions were not due to malarial nephropathy, and the disease was different from the minimal change nephrotic syndrome. In this same study it was found that $75 \%$ of the Indian children from the same region had minimal change nephrotic syndrome that was responsive to steroids, and the disease was similar to that seen in other parts of the world (7).

A study from the United Kingdom reported that the incidence of minimal change nephrotic syndrome was six fold higher in Asian children born in the U.K. when compared with the English children from the same region. The pattern of the disease however due to minimal change in the Asian children has been reported to be similar to that seen in the West (8).

In Sri Lanka too, nephrotic syndrome is commonly seen and the clinical features and the response to steroids appear to be similar to that 
reported from the West and other Asian countries. We have observed however that there is a high relapse rate among our children. These children have had repeated courses of steroids and most of them developed signs of steroid toxicity needing alternative drug therapy (9).

Apart from a brief report of 17 renal biopsies done on children with nephrotic syndrome (10) there are no published studies of the histopathology of the kidneys in children with nephrotic syndrome from Sri Lanka.

In a cohort of 63 children admitted to the Teaching Hospital, Peradeniya with primary nephrotic syndrome, percutaneous renal biopsies were performed in children who had frequent relapses and those with atypical features.

This is a preliminary report of an ongoing study of the histopatholodical features seen in the kidneys in this selected group of patients.

An attempt is also made to correlate the clinical features of these patients with the morphological changes in the kidneys.

\section{Patients and Methods}

The study group consisted of 63 children less than 15 years of age with an age range of four to fourteen admitted to the Teaching Hospital, Peradeniya from 1982 to date. Ŕanal biopsies were performed from January 1993 onwards on 22 of these patients.

The indications for renal biopsies were as follows:

1. Children who initially responded to steroid therapy but had frequent relapses (Two relapses with in six months or more than three relapses in one year) and were steroid dependant.

2. Patients with macroscopic or persistent microscopic haematuria lasting over one month.

3. Patients with persistent hypertension with a diastolic pressure of over $90 \mathrm{~mm} \mathrm{Hg}$ lasting over one month.

4. Children who were resistant to steroid therapy from the onset of the illness.
Percutaneous renal biopsies were done under midazolam sedation using a Tru-cut biopsy needle.

Renal tissue was fixed in $10 \%$ formol saline for a minimum of 24 hours and processed into paraffin.

The initial paraffin sections were stained with haematoxylin and eosin, methenamine silver stains for basement membranes, periodic acid Schiff, Van Gieson and Masson's trichrome stains.

The next set of paraffin sections were stained for IgM, IgG, IgA and C 3 using polyclonal antibodies, and a three stage streptavidin biotin peroxidase immunohistochemical staining system. Digestion with $0.05 \%$ protease for 3 minutes was carried out before application of the primary antibody. The chromogen used was 3'3 diaminobenzidine tetrahydrochloride. A further series of paraffin sections were cut and stained with haematoxylin and eosin thereafter. All sections were viewed with the light microscope. No electron microscopic studies were done.

Three children who had nephrotic syndrome secondary to systemic lupus erythematosus and one child who had diabetic nephropathy are not included in this report.

\section{Results}

The histopathological findings of the renal biopsies are shown in Table 1.

Table 1

Pathological changes observed in the patients with nephrotic syndrome subjected to percutaneous renal biopsy

Histopathological changes $\quad$ No. of patients

1. Minimal change lesion 9

2. Focal segmental glomerulosclerosis $\quad 7$

3. Membranous glomerulonephritis 3

4. Mesangio-capillary
glomerulonephritis

5. Diffuse mesangial proliferation

9




\section{Minimal Change lesion}

The renal tissue showed normal glomeruli. Occasionally, a mild increase in the mesangium was seen. Immune deposits were not detected.

\section{Focal segmental glomerulosclerosis}

Some glomeruli, showed segmental lesions. These included, segmental collapse of capillaries and sclerotic lesions away from the vascular pole, tuft adhesions, and lesions of hyalinosis in the segmental lesions (Figures 1A \& 1B). In addition, sclerotic glomeruli and mild mesangial hypercellularity and widening were observed. Tubular atrophy with associated interstitial inflammation were seen in association with sclerotic glomeruli.

The diseased glomeruli showed deposits of IgM in the sclerotic region. $\operatorname{IgM}$ was also demonstrated occasionally in the mesangium in unaffected glomeruli, occasionally (Figure 1C).
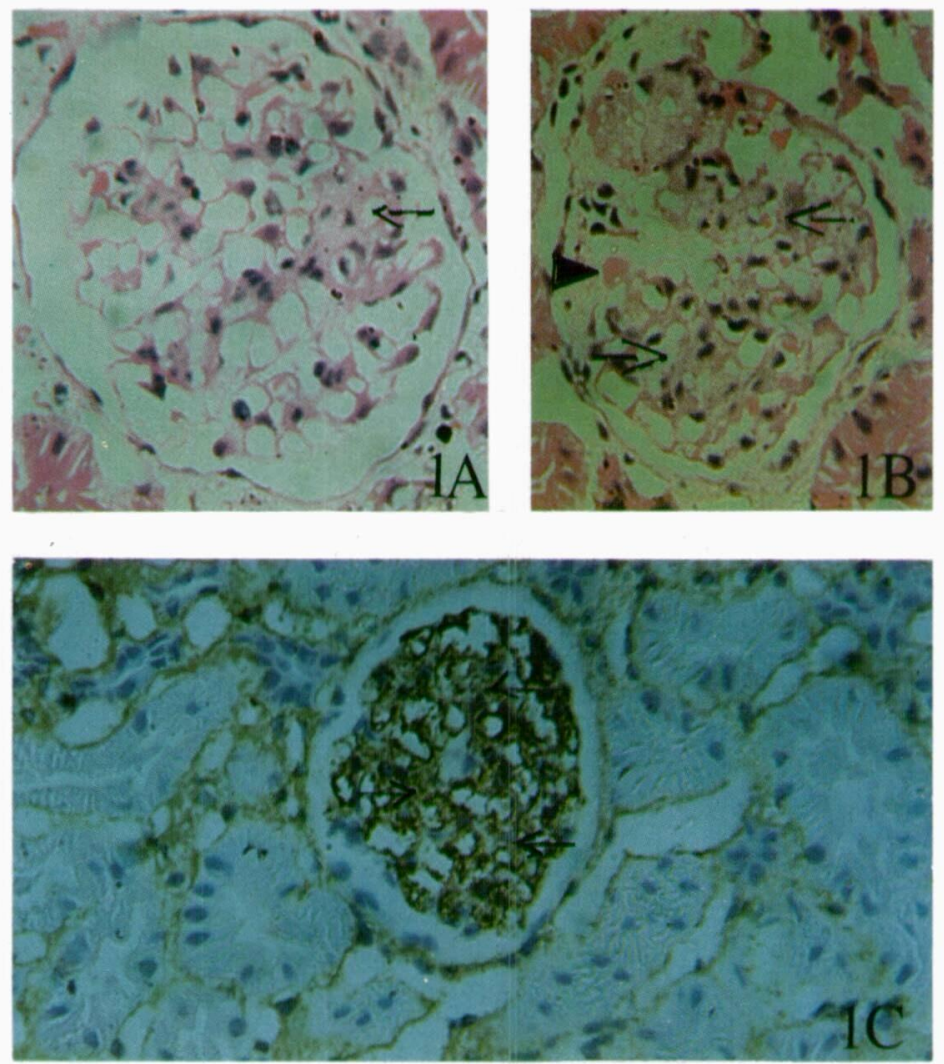

Fig: 1A A glomerulus showing a segmental sclerotic lesion-arrow. The rest of the capillaries are normal.

Haematoxylin and eosin $\times 240$.

Fig. 1B A glomerulus showing two segmental lesions - arrows. A hyaline globule is seen in one lesion - arrow head

Haematoxylin and eosin $\times 240$

Fig: 1C Normal glomerulus showing coarse granules of IgM in the mesangium staining brown arrow.

Streptavidin - Biotin Peroxidase $x 240$. 


\section{Membranous glomerulonephritis}

The glomeruli showed diffuse thickening of the basement membrane of the peripheral capillary wall. The silver stains revealed a 'spike' like deposition of basement membrane material and also vacuolation of the thickened membrane. Granular epimembranous deposits of IgG and C3 were seen with immunostaining. A small amount of IgA was also seen.

\section{Mesangio-capillary glomerulonephritis}

The glomeruli showed an increase in the mesangium, and thickening of the peripheral capillary wall. The latter showed a double contouring with the silver stains.

\section{Diffuse mesangial proliferation}

All glomeruli showed a widening of the mesangium, and an increase in the mesangial cellularity. Immuno-staining was negative for IgA but focal mesangial IgG deposits were seen.

The glomerular pathology was correlated with the age, sex, and the presence of hypertension and/or haematuria in these patients. The distributions of the histological lesions according to the age at onset of the nephrotic syndrome is shown in Figure 2.
It was observed that six out of a total of nine children with minimal change histopathology were five years or less at the onset of the disease. Except for the group of children with focal segmental glomerulosclerosis, all the other patients with significant structural histopathology were aged seven years or more.

The distribution by sex of the glomerular pathology is shown in Table 2.

In our study it was observed that there are more females than males in the minimal change category.

\section{Haematuria and Hypertension}

The relationship between persistent haematuria and hypertension and glomerular pathology is shown in Table 3.

In the group with minimal change was a ten year old girl who had intractable hypertension.

Both children who had mesangiocapillary glomerulonephritis were female, aged twelve and eight and a half years. Both presented with haematuria and hypertension at the onset of the illness.

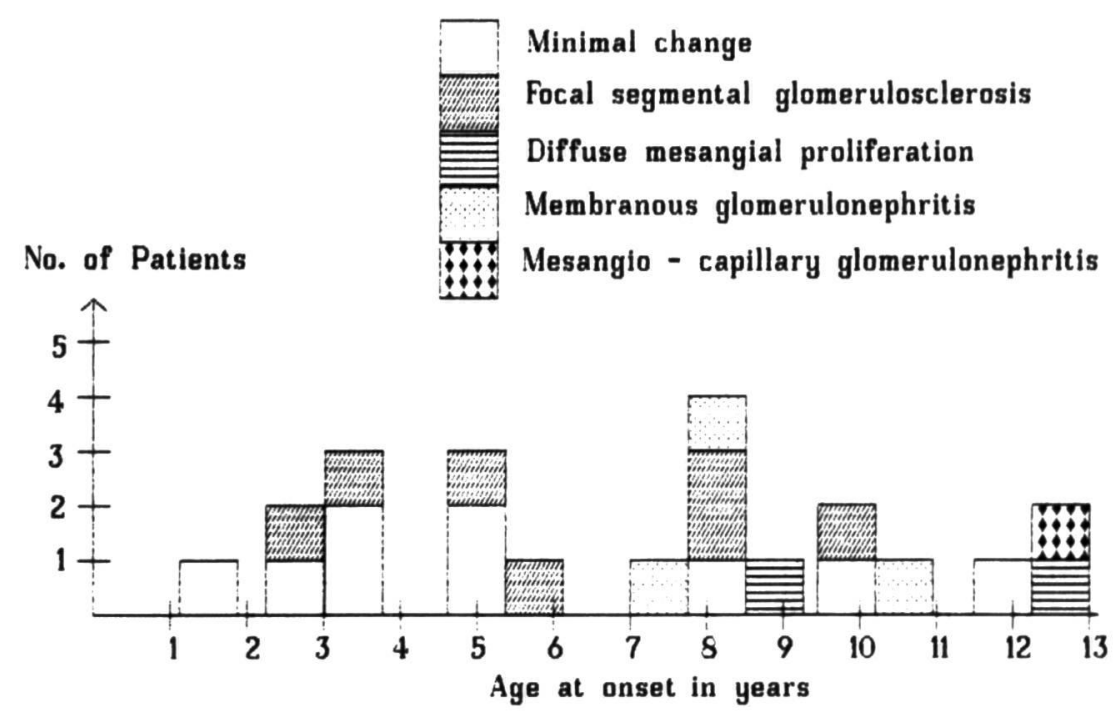

Figure 2: Distribution of the histological lesions according to age at onset of the nephrotic syndrome 
Table 2

Glomerular pathology related to the sex of the patient

\begin{tabular}{lccc}
\hline Glomerular Pathology & No. of patients & Male & Female \\
\hline Minimal change & 9 & 3 & 6 \\
Focal segmental glomerulosclerosis & 7 & 6 & 1 \\
Membranous glomerulonephritis & 3 & 2 & 1 \\
Mesangiocapillary glomerulonephritis & 2 & - & 2 \\
Diffuse mesangial prolliferation & 1 & 1 & - \\
\hline
\end{tabular}

Table 3

Haematuria and/or hypertension at onset of the disease related to glomerular pathology

\begin{tabular}{lccc}
\hline Glomerular Pathology & No. & Haematuria & Hypertension \\
\hline Minimal change & 9 & - & 1 \\
Focal segmental glomerulosclerosis & 7 & 1 & - \\
Mesangiocapillary glomerulonephritis & 2 & 2 & 2 \\
Membranous glomerulonephritis & 3 & - & - \\
Diffuse mesangial prolliferation & 1 & 1 & - \\
\hline
\end{tabular}

\section{Discussion}

13 children out of 22 on whom renal biopsies were performed had significant structural changes. Of these patients the largest group of seven children was classified under the histopathological category of focal segmental glomerulosclerosis. Focal segmental glomerulosclerosis is now a well recognised entity and is believed to have a poor prognosis. It may be difficult to differentiate this from minimal change nephrotic syndrome in the early stages. This lesion occurs initially in the juxtamedullary glomeruli and then spreads to the rest of the cortex. The lesion could be missed in a renal biopsy due to its focal nature. It has been documented that where the initial biopsy was reported to be of the minimal change type, the subsequent biopsy had demonstrated focal segmental glomerulosclerosis (4).
There were six female children out of a total of nine in the group with minimal change nephrotic syndrome. This is in marked contrast to the usual male preponderance that is seen. It is possible that some of these patients may ultimately develop focal segmental glomerulosclerosis. The ten year old female patient who presented with intractable hypertension at the onset of her illness may also present later with focal segmental glomerulosclerosis.

Patients with mesangio-capillary lesions are believed to apparently recover inspite of being unresponsive to steroids. It is too early to comment on our patients.

Knowledge of the underlying pathology will help the clinician in the management of the patient and also gives an indication of the 
prognosis of the condition. Hence a renal biopsy is mandatory in the children who are difficult to treat, or who have atypical features.

Further studies are needed especially from the Asian region as it has been shown that the incidence of nephrotic syndrome is higher in Asian children than those from the West.

It is important to ascertain whether race, poor socio-economic conditions leading to repeated infections or any other factors play a role in the pathogenesis of this disease. This is being investigated in our long-term ongoing study.

\section{Acknowledgements}

We thank all the doctors, nurses and the medical students who looked after these children; Miss S. Ramadasa, Technician, Dept. of Pathology for preparation of the histopathology and immunostaining slides and Gamini Gunasekere for the photographs; Mrs. Padmalatha Menike Rajapakse for typing the script.

\section{References}

1. Srivastava RN, Mayekar G, Anand R, Choudhry VP, Ghai OP, Tandon HD. Nephrotic syndrome in Indian children. Archives of Disease in Childhood 1975; 50: 626-630.

2. Elzouki AY, Amin F, Jaisawal OP. Primary nephrotic syndrome in Arab children. Archives of Disease in Childhood. 1984; 59: 253-255.
3. Churg J, Habib R, White RHR. Pathology of the nephrotic syndrome in children. Lancet 1970; 1: 1299-1302.

4. White RHR, Glasgow EF, Mills RJ. Clinicopathological Study of Nephrotic Syndrome in Childhood. The Lancet 1970; 1: 1353-1359.

5. Koskimies O, Vilska J, Rapola J, Hallman N. Long-term outcome of primary nephrotic syndrome. Archives of Disease in Childhood. 1982; 57: 544-548

6. Hendrickse RG, Adeniyi A, Edington GM, Glasgow EF, White RHR, Houba V. Quartan malarial nephrotic syndrome: collaborative clinico-pathological study in Nigerian children. Lancet 1972; 1: 1143-1148.

7. Coovadia HM, Adhikari M, Morel-Maroger L. Clinico-pathological features of the nephrotic syndrome in South African children. Quarterly Journal of Medicine. New Series XLVIII 1979; 189: 77-91.

8. Feehally J, Kendell NP, Swift PGF, Walls J. High incidence of minimal change nephtrotic syndrome in Asians. Archives of Disease in Childhood, 1985; 60: 1018-1020.

9. Jayasena L, Abeygunawardena AS. Levamisole in childhood nephrotic syndrome. Kandy Medical Journal 1994; 3: $15-16$.

10. Soysa P. Effective care of the child. Ceylon Journal of Child Health 1973; 8: 3-14. 